\title{
La crisis de identidad de las cooperativas agrarias en Francia
}

\author{
Amanda Vargas-Prieto
}

* Doctora en Ciencias Económicas, Universidad de Burdeos, Francia. Profesora asociada, Facultad de Ciencias Económicas y Sociales, Universidad de La Salle, Bogotá, Colombia.

Correo electrónico: amvargas@unisalle.edu.co

Recibido: 12 de septiembre del 2014 Aprobado: 7 de noviembre del 2014

Cómo citar este artículo: Vargas-Prieto, $A$. (2014). La crisis de identidad de las cooperativas agrarias en Francia. Cooperativismo \& Desarrollo, 22(105), 31-46. doi: http://dx.doi.org/10.16925/co.v22i105.1032

\section{Resumen}

El contexto evolutivo del sector agrícola en Francia llevó a las cooperativas agrarias a crear grupos de empresas que combinan las cooperativas tradicionales - encargadas del proceso de producción-y las filiales, encargadas de agregar valor a la producción. En este artículo se presenta la especificidad de los grupos cooperativos agrarios en Francia. La nueva estructura cooperativa lleva a asociar el modo de gobernanza asociativo de la estructura tradicional y el modo de gobernanza accionarial de las filiales en un modo híbrido que provoca una crisis de identidad a este tipo de empresa.

Palabras clave: agricultura, cooperativas, cooperativas agrarias, Francia, gobernanza.

\section{The Identity Crisis of Agrarian Cooperatives in France}

\begin{abstract}
The evolutionary context of the agrarian sector in France led the agrarian cooperatives to create groups of companies that combine the traditional cooperatives, responsible for the production process, with subsidiaries to add value to production. This article shows the specificity of the agrarian cooperative groups in France. The new cooperative structure leads to a connection between the associative governance mode of the traditional structure and the equity governance mode of the subsidiaries in a hybrid mode that gives rise to an identity crisis for this type of company.
\end{abstract}

Keywords: agriculture, cooperatives, agrarian cooperatives, France, governance.

\section{A crise de identidade das cooperativas agrárias na França}

\section{Resumo}

O contexto evolutivo do setor agrícola na França levou as cooperativas agrárias a criarem grupos de empresas que combinassem as cooperativas tradicionais - encarregadas do processo de produção- $\mathrm{e}$ as filiais - encarregadas de agregarem valor à produção-. Neste artigo, apresenta-se a especificidade dos grupos cooperativos agrários na França. A nova estrutura cooperativa leva à associação do modo de governança associativa da estrutura tradicional e do modo de governança acionista das filiais num modo híbrido que provoca uma crise de identidade nesse tipo de empresa.

Palavras-chave: agricultura, cooperativas, cooperativas agrárias, França, governança. 


\section{Introducción}

El sector de la cooperación agrícola en Francia se organizó principalmente bajo la influencia de tres grandes leyes (CFCA, 1999): la política agrícola de 1960 y 1962 sobre la organización económica de los productores, y la ley de 1972 sobre el estatus jurídico de la cooperación agrícola. Tras el endurecimiento del entorno competitivo agrícola en Europa, vinculado a la globalización, las leyes de 1991 y 1992 se adoptan con el fin de organizar las filiales y el fortalecimiento de los fondos propios de las cooperativas agrarias (Eurostaf, 2007). Estas últimas son responsables del desarrollo acelerado de los grupos empresariales cooperativos en la decada de 1990 (Forestier y Mauget, 2000). De esta manera, estos grupos se han posicionado en el mercado agroalimentario europeo gracias a la combinación de cooperativas tradicionales y filiales, las cuales son empresas privadas en aval de la producción. La dualidad de los modos de gobernanza (asociativo y accionarial) de estos grupos cooperativos puede ser representada por un modo híbrido. En este artículo, se expone cómo este tipo de gobernanza híbrida provoca una crisis de identidad, ya que no deja de tener consecuencias en la relación de la cooperativa con sus asociados ${ }^{1}$. Esta crisis se manifiesta con tres efectos negativos: el distanciamiento, la complejidad y la confusión.

El artículo está organizado en tres partes que, en conjunto, buscan caracterizar la gobernanza de las cooperativas agrarias en un contexto evolutivo. A través de una revisión de la literatura sobre las cooperativas agrarias, analizamos su evolución estructural desde la aparición de pequeñas cooperativas tradicionales hacia los grandes grupos cooperativos, para luego entender las consecuencias de su evolución estructural en su gobernanza. Finalmente, se presentan los efectos negativos en la relación cooperativa-asociados de este cambio en la gobernanza, explicando así la crisis de identidad de las cooperativas agrarias.

\section{La especificidad de la gobernanza de las cooperativas agrarias en Francia}

\section{De la pequeña cooperativa hacia los grandes grupos cooperativos}

Alrededor del mundo, todas las empresas vivieron profundos cambios a fin de mantener un lugar en esta eco-

1 Se hace uso del término asociados para designar los socios, asociados o adherentes. nomía global, en la cual la competencia aumenta de manera exponencial. Las cooperativas no escapan de esta tendencia. Para asegurar su supervivencia y aumentar su eficiencia, las cooperativas agrarias han cambiado su estructura organizacional, en particular a través de fusiones y adquisiciones (Bélis-Bergouignan y Corade, 2008; Forestier y Mauget, 2000; SaintPierre, 2004). Con el objetivo de mantener su cuota de mercado, las cooperativas en Francia se desarrollaron adoptando estrategias similares a sus competidores del sector privado, esto es, diversificando sus actividades hasta constituir los grandes grupos cooperativos a través de holdings financieros ${ }^{2}$ (Forestier y Mauget, 2000). La adopción de esta nueva forma de empresa cooperativa debe ser estudiada para demostrar sus desafíos y comprender los impactos de su comportamiento. Se trata entonces de identificar los factores de la evolución de las cooperativas agrarias francesas y así entender el surgimiento de grupos de cooperativas agrarias en Francia. A continuación se presenta una tipología de las cooperativas agrarias resultantes de este desarrollo.

\section{Factores de evolución de las cooperativas agrarias en Francia}

Desde 1960, las grandes superficies se posicionaron significativamente en la industria agroalimentaria francesa (Deneux, Bizet y Dussaut, 1999). Este posicionamiento llevó a la creación de centrales de compra, es decir, organizaciones que tienen el objetivo de reagrupar las compras de las organizaciones afiliadas con el fin de reducir los costos. La presión creada por las centrales de compras obligó a las empresas del sector a buscar un tamaño importante para estar en igualdad de condiciones en las negociaciones. Así, las empresas menos competitivas han desaparecido y grandes grupos de empresas agroalimentarias han surgido en el sector agrícola francés.

Por lo tanto, las cooperativas agrarias francesas ubicadas en todo el territorio ${ }^{3}$ han decidido unirse, creando así grandes grupos cooperativos, como InVivo, Terrena, Tereos, Sodiaal, Champagne-Céréales, Agrial y Axéréal, entre otros, con el objetivo de flexibilizar la estructura cooperativa tradicional.

\footnotetext{
2 Un holding es una entidad creada dentro de un grupo de empresas con el fin de tener acciones en otras empresas del grupo y así cierto poder estratégico (Charreaux, 1997; Manoa, 2001; OCDE, 2001).

3 Esta repartición es, sin embargo, desigual. Según Duchamp y Mauget (2012), las zonas con una presencia importante son: la región de Burdeos, el sur este y la Marne (viticultura). Sin embargo, si se consideran los ingresos, ellas son originarias del oeste de Francia.
} 
La constitución de estos grupos se produce de varias maneras (Koulytchizky y Mauget, 2002):

1. A través de fusiones (la alianza más exitosa entre las cooperativas suelen ser las uniones), o la unión de cooperativas de comercialización o la unión por el manejo de una marca (por ejemplo, Even se unió con Coopagri Bretagne y Cana en la marca Paysan Breton (productos lácteos).

2. La creación de grupos de transformación y de comercialización con una actividad dominante (la unión nacional de cooperativas de cereales - UNCAC).

3. Creando grupos polivalentes (a través de cooperativas centralizadas o de uniones como UNICOPA).

Además de este contexto competitivo a nivel nacional, las cooperativas debieron actuar ante la globalización. Koulytchizky y Mauget (2001) sintetizan los factores de cambio en cinco puntos clave que permiten explicar por qué las cooperativas realizaron las diferentes maniobras estratégicas que participaron en la creación de los grupos cooperativos. Los cinco puntos claves son: 1) la europeización y la globalización de los mercados y de la competencia, 2) la internacionalización de las grandes superficies y las estrategias de descuento, 3) el crecimiento de la demanda de productos transformados, 4) las nuevas normas reglamentarias y 5) la evolución del comportamiento de los agricultores.

Después de la firma del acta única y de los acuerdos entre la General Agreement on Tariffs and Trade (GatT) y la Organización Mundial del Comercio (омc), las cooperativas debieron hacer frente a la europeización y a la globalización de los mercados y de la competencia. La estrategia de cambio de la dimensión del mercado fue compleja y necesitó una cantidad importante de medios financieros. De hecho, la globalización de los mercados favorece a las empresas privadas, las cuales movilizan más fácilmente su capital (Spear, 2001). La evolución de las grandes superficies tuvo también un impacto importante sobre las cooperativas, ya que la internacionalización de las grandes superficies y las estrategias de descuento obligaron a las cooperativas a valorizar la producción de sus asociados. Las empresas cooperativas, a causa de límites en los estatutos, no pueden implantarse en el extranjero fácilmente. La demanda creciente de productos transformados - debida a la evolución de la demanda de los consumidores - tiene relación con su interés por la salud y la nutrición (por ejemplo, la disminución del consumo de productos cárnicos, de vinos, de azúcar, etc.). Luego, las nuevas normas influenciaron el contexto agrícola. Así, la liberación de los mercados tiene una influencia directa sobre las normas nacionales que, antes, protegían las cooperativas francesas (Spear, 2001). Además, la obligación de estar al día en las nuevas normas conduce a inversiones muy altas para las pequeñas cooperativas. Por ejemplo, las reformas sucesivas de la Política Agrícola Común (PAC) han dado lugar a pagos más bajos a los agricultores. Estas reformas han afectado principalmente a las cooperativas de cereales, las cuales controlan alrededor del $70 \%$ de la colecta en Francia. Finalmente, el último factor de cambio - quizás el más importante-, ya que ha dado lugar a la evolución de los valores cooperativos, es la evolución del comportamiento de los agricultores. Estos están mejor capacitados, más y mejor informados, son más exigentes (Eurostaf, 2007) y tienen más dificultades financieras (Colson, 1990) y técnicas (problemas relacionados con la distribución y comercialización del producto). Sus expectativas son altas y han cambiado con respecto a lo que existía en el momento de la creación de las cooperativas.

Ahora, en el contexto de la globalización, con el fin de asegurar su posición de liderazgo en los mercados mundiales, las cooperativas agrarias se ven obligadas a repensar sus estrategias y desarrollar estrategias de internacionalización (Eurostaf, 2007). Para simplificar el análisis, optamos por unir los puntos clave 1 y 2 de Koulytchizky y Mauget (2001) en un solo factor de cambio que denominamos internacionalización y globalización de los mercados y los competidores. En relación con los puntos clave 3,4 y 5 , es posible asociar las consecuencias de estos cambios y deducir los retos para el sector cooperativo. Un segundo factor de cambio es entonces la evolución del consumo en Francia. El cambio en los hábitos alimenticios de los franceses exigió un gran esfuerzo de adaptación por parte de las cooperativas agrarias. Esta adaptación demandó importantes recursos financieros para la investigación y el desarrollo, la maquinaria agrícola y la publicidad. Las cooperativas tuvieron que elegir entre ser productoras de materias primas y comercializarlas a los grandes grupos privados, o crear estrategias de desarrollo mediante la inversión en las filiales de transformación dentro de su propia estructura. El tercer factor de cambio es la evolución de las limitaciones reglamentarias. La disminución de las medidas de protección a favor de los agricultores, la necesidad de cumplir con nuevas normas y la exigencia de respetar un desarrollo 
sostenible dio lugar a una inversión excesiva para las cooperativas agrarias. Su objetivo ahora es producir más y mejor, y con menos insumos químicos. Por último, el cuarto factor de cambio es la evolución del comportamiento de los agricultores. Los agricultores están exigiendo cada vez más la calidad del contenido y de los servicios prestados por las cooperativas. Esto está relacionado con los cambios de valores de los grupos cooperativos. Los agricultores sienten la multiplicidad y variedad de sus diferentes necesidades, de sus proyectos y de sus expectativas. Es por eso que ahora esperan asesoramiento más personalizado. Además, el nivel de competencia técnica de los agricultores es alto, $\mathrm{y}$, por lo tanto, están directamente involucrados en la asesoría con el fin de optimizar el tiempo de esta, pues si la asesoría es adaptada a sus necesidades puede ser fuente de valor agregado. Por ello, algunos agricultores reclaman una adaptación y una mayor diversificación de los servicios ofrecidos por los organismos de asesoría agrícola. Cuando se implementa la mejora solicitada y la diversificación de los servicios, el organismo de asesoría muestra la capacidad para adaptarse y ofrecer un servicio personalizado, lo que se convierte en un valor agregado que permite ser más competitivo (Vargas-Prieto, 2013). Los factores de cambio, sus consecuencias y desafíos para las cooperativas agrarias se resumen en la tabla 1 (ver Anexo).

\section{La aparición de grupos cooperativos agrarios en Francia}

En respuesta a estos factores de cambio, las cooperativas agrarias desarrollaron varios tipos de maniobras estratégicas (Koulytchizky y Mauget, 2001; Koulytchizky y Mauget, 2002; Eurostaf, 2007).

En primer lugar, la estrategia de integración horizontal se logra mediante la concentración de la oferta, con el fin de recuperar la cuota de mercado. Muchas cooperativas han utilizado esta estrategia en los últimos años. Para ello se han realizado concentraciones de dos maneras:

1. A través de la agrupación de cooperativas como lo hicieron algunos de los grupos que estudiamos.

2. A través de la compra de empresas privadas. La concentración de actividades permite reducir los costos y mejorar la eficiencia (colecta, herramientas industriales, comercialización e investigación).
Las cooperativas son entonces más competitivas en comparación con los contratos de la competencia (precios al productor) y servicios, lo que permitiría recuperar la cuota de mercado. La integración horizontal también aumentó la polivalencia, es decir, la diversificación de las actividades dentro de una sola estructura. Hoy en día, el modelo de gran cooperativa polivalente es una especificidad francesa, en comparación con el resto de Europa, donde las cooperativas son especializadas en un solo tipo de producción (Cogeca, 2011).

Una segunda estrategia (la integración vertical) tiene como objetivo crear economías de escala mediante la integración de las filiales transformadoras. La integración vertical se desarrolló principalmente en el sector de producción animal (leche y carne), debido a la complejidad del sector (necesidad de estructuras de colecta de leche y mataderos cerca del lugar de producción). Sin embargo, la integración vertical permitió un crecimiento interno acompañado por un crecimiento externo, a través de alianzas o de fusiones y adquisiciones de empresas no cooperativas. En Francia, los grandes grupos cooperativos agrarios comienzan a internacionalizarse mediante el uso de herramientas industriales, centros de investigación, procesamiento y circuitos de distribución en el extranjero.

Tras el endurecimiento del entorno competitivo agrícola que supuso la globalización de los mercados y el hacer frente a las grandes empresas privadas - las cuales no se limitan en términos geográficos-, las leyes de 1991 y 1992 organizaron el proceso de creación de filiales. Esto permitió a las cooperativas agrarias francesas, a través de la formación de grupos, aumentar su tamaño y su cuota de mercado (Eurostaf, 2007) . $^{4}$ Según Fort y Couderc (2001), el tamaño aparece como un factor importante de desarrollo para las empresas del sector agroalimentario. Además, la filial permite el fortalecimiento de los fondos propios de las cooperativas (Nicolas, 1992; 1993) para el desarrollo de grandes grupos cooperativos. El resultado de estas maniobras estratégicas se reflejó en la aparición de grandes grupos cooperativos que combinan un estatus cooperativo y filiales privadas. Por lo tanto, la creación de grupos cooperativos agrarios permite evadir las restricciones estatutarias de las cooperativas y el desarrollo de la transformación y comercialización (Filippi, 2002). Según varios autores, los grupos cooperativos se van a convertir en el modelo de referencia en el sector

\footnotetext{
4 El tamaño es considerado un factor clave de éxito por varios autores (Cogeca, 2011; Rouault, 2010).
} 
agrícola (Filippi, 2002; Forestier y Mauget, 2000; Guillouzo, Perrot y Ruffio, 1999; Nicolas, 1992; 1993). Según Coop de France ${ }^{5}$, el sector cooperativo representa el 40\% del sector agroalimentario en Francia y empleó a más de 160000 empleados en el 2009 (Coop de France, 2010). De hecho, durante el período 1995-2005, el perímetro cooperativo creció alrededor del $50 \%$ en términos de número de empleados, con el $84 \%$ de los empleados trabajando en un grupo cooperativo en 2005 (Lesieur, 2012). El posicionamiento de los grupos cooperativos permitió el control de la industria agroalimentaria a través de un proceso de concentración a través de las filiales ${ }^{6}$ con el fin de alcanzar un tamaño importante (industrial, comercial, de investigación y financiero), y de esta manera entrar en mercados internacionales (Guillouzo et al., 1999). Pero si los grupos cooperativos son actualmente el modelo de referencia en Francia, se organizan de manera diferente en función de su estrategia de crecimiento.

\section{Diversidad de las cooperativas agrarias en Francia}

A raíz de estos acontecimientos, el sector agrícola cooperativo se compone actualmente de cinco tipos de sociedades. Esta clasificación se basa en dos criterios específicos, los cuales se encuentran en los nombres de los tipos: el perímetro territorial de la colecta, es decir, la zona de influencia de la cooperativa (nacional, regional, departamental, local); y la naturaleza de la actividad, polivalente o especializada (Eurostaf, 2007). Así, podemos determinar cinco tipos de sociedades cooperativas, de los cuales cuatro son grupos cooperativos: grupos nacionales especializados, grupos regionales especializados, grupos regionales polivalentes, grupos departamentales polivalentes y cooperativas locales.

En primer lugar, las cooperativas locales son el primer tipo de sociedades cooperativas; incluyen pequeñas cooperativas y están en un nivel municipal o distrital ${ }^{7}$. Las pequeñas cooperativas son las más tradicionales y se crean con el fin de reunir la oferta de productos geográficamente localizados y así determinar un precio mínimo para el asociado. La naturaleza

\footnotetext{
5 Federación Nacional de Cooperativas Agrarias en Francia.

6 El top 20 de los grupos cooperativos agrarios franceses pasó de 30\% de los ingresos en el 2008, a 46\% en el 2010 (Duchamp y Mauget, 2012).

$7 \quad$ Según las encuestas anuales del sector, las pequeñas cooperativas son definidas como empresas de diez o menos empleados y no pasan el limite de cinco millones de euros de ingresos en el sector agroalimentario, y 38 millones de euros en el sector de mayoristas (Insee, 2010).
}

de sus actividades es variable - por ejemplo, las cooperativas de frutas y verduras del este de Francia-, o especializada - por ejemplo, las pequeñas cooperativas vitícolas representan $85 \%$ de la industria de bebidas (Magrini, Fares y Filippi, 2011)-. Especialmente, se encuentran pequeñas cooperativas en la industria del vino, productos lácteos y cereales. Los otros cuatro tipos de cooperativas son grupos y resultan de particular interés si se tiene en cuenta su importancia en el sector.

El segundo tipo consiste en los grupos departamentales polivalentes. Están formados por cooperativas que se unen para comprar suministros y la distribución de los productos. El tercer tipo incluye los grupos regionales polivalentes, los cuales tienen las mismas características que el anterior, pero se encuentran en un territorio mucho más amplio, es decir, varios departamentos o varias regiones. Estos grupos tratan de aumentar su dimensión industrial y comercial intensificando las agrupaciones (alianzas), así como para ampliar su dimensión geográfica, pasando a un nivel regional o nacional (Eurostaf, 2007). El cuarto tipo consiste en grupos regionales especializados. Estos abarcan hasta tres o cuatro regiones. Según los autores del estudio Eurostaf (2007), este tipo de grupo tiende a crecer, a ser nacional o incluso internacionalizarse. Por último, el quinto y último tipo se compone de grupos nacionales especializados. Estos grupos tienen una cobertura nacional. Ellos son considerados el modelo principal de la sociedad cooperativa agrícola en términos de tamaño. Este modelo ganará importancia en los próximos años debido al aumento de la competencia (Eurostaf, 2007).

Entre estos cinco tipos de sociedades cooperativas, es posible distinguir una diversidad de formas de organización. El resultado de las maniobras estratégicas que crearon las sociedades cooperativas se traduce en cuatro formas organizativas distintas, según la exposición de Filippi, Frey, Triboulet y Vivensang (2006) en la figura 1.

La forma 1 representa las cooperativas independientes, a las que nos hemos referido como locales (lo cual corresponde al primer tipo de sociedad cooperativa). Las formas 2, 3 y 4 corresponden a los grupos cooperativos (tipos 2 a 5: departamentales, regionales o nacionales y especializados o polivalentes). La elección de la estructura está claramente relacionada con la estrategia elegida por la sociedad cooperativa para la creación del grupo (Chandler, 1989). La forma 2 corresponde a los grupos que han optado por controlar directamente sus filiales. Según Filippi et al. (2006), la 


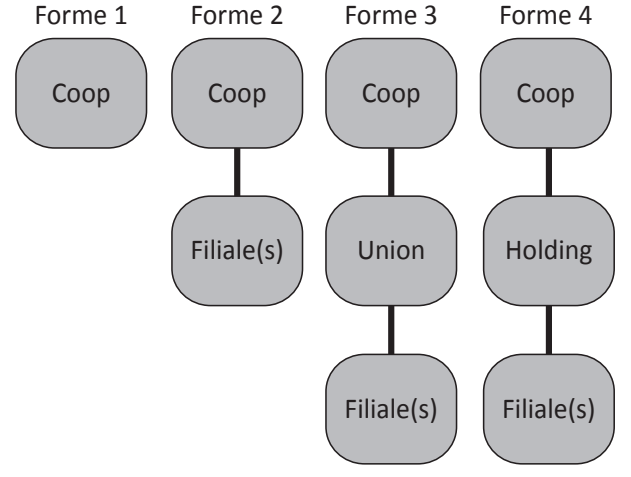

Figura 1. Formas de organización de las cooperativas agrarias. Tomado de Bilan des Lois de 1991 et 1992 et Gouvernance des Groupes Coopératifs, por Filippi et al., 2006, p. 72.

forma 2 es la dominante en Francia. La forma 3 corresponde a los grupos que se han formado por la unión de varias cooperativas y, en este caso, es la unión que controla las filiales. Por último, la forma 4 representa las cooperativas que crearon una sociedad holding de tipo s. A. En ese caso, las filiales son controladas por la holding. Este tipo de grupos está en su mayoría presente en las cooperativas de cereales. Según Duchamp y Mauget (2012), la mayoría de grupos cooperativos del top 20 francés eligieron la forma 4 , de manera que interviene un holding financiero en su estructura.

Hasta ahora, se ha expuesto cómo el contexto ha llevado a las cooperativas a desarrollar estrategias que han hecho surgir grupos cooperativos en el sector agrícola. Estos grupos cooperativos están formados por cooperativas tradicionales y filiales de transformación, lo cual conduce a un modo específico de gobernanza. En ese orden de ideas, hay una mezcla de modos de gobernanza asociativo y accionarial que cuestiona la identidad de las cooperativas. La siguiente sección presenta el modo híbrido de gobernanza que se ha desarrollado en los grupos cooperativos y se deriva de estos dos modos de gobernanza.

\section{El modo de gobernanza híbrido de los grupos cooperativos agrarios}

En un principio, la gobernanza de las cooperativas era considerada original porque se basaba en el principio de la democracia. Según los principios originales de las cooperativas, los asociados están involucrados en la selección de los dirigentes, el control y la gestión, y también en la elección de la estrategia de la cooperativa. Este compromiso se refleja en el principio de "un hombre, un voto", el cual, según la ley cooperativa, garantiza el equilibrio de poderes: cada miembro de la cooperativa tiene derecho a votar, independientemente del tamaño de su explotación (Ley de 4 de septiembre 1943). Sin embargo, la evolución de las sociedades cooperativas - marcada por la aparición de grupos-, cuestiona, al menos en parte, estos principios de funcionamiento. Por tanto, esta sección analiza a través del concepto de gobernanza los dos modos opuestos dentro de los grupos cooperativos.

Primero, se define el concepto de gobierno corporativo, con el fin de entender cómo la aparición de grupos de cooperativas agrarias en Francia llevó a la mezcla de dos modos diferentes de gobernanza. Luego, se verá cómo la evolución de las cooperativas se ha traducido en el paso de un modo de gobernanza asociativo a un modo de gobernanza accionarial. Este modo intermedio es denominado modo híbrido de gobernanza.

\section{Las dos grandes visiones del gobierno corporativo}

Para Charreaux (1997), la gobernanza se refiere a los mecanismos que delimitan de manera efectiva el poder de los dirigentes y de influir en sus decisiones. Uno de los primeros análisis que busca entender quién en realidad controla la empresa y de qué manera es el de Berle y Means (1932). La literatura atribuye a estos autores la aparición del concepto de gobierno corporativo. Para ellos, el problema de la crisis de 1929 nació a partir de la ruptura de la noción de propiedad. Según Charreaux (2000): “(...) el papel de supervisión de los accionistas, que implica sistemas de incentivos y de vigilancia y un papel en la toma de decisiones por parte de los directivos" (p. 2). Su objetivo era mostrar que el desarrollo de una sociedad por acciones genera la separación de la propiedad y el control de la empresa. El poder de decisión pasa entonces de los accionistas-propietarios de la empresa, a los directores a cargo de la gestión de esta (Plane, 2000). La gobernanza surge de la necesidad de vigilar la gestión de los dirigentes y definir las reglas del juego, en cuanto a la delegación de gestión y control del poder en la empresa y el proceso de toma de decisiones (Charreaux, 2000; Baron, 2003).

Las teorías de la gobernanza tienen dos visiones principales que ofrecen diferentes explicaciones sobre la eficiencia de las organizaciones en términos de creación y distribución de valor: la visión contractual de la empresa y la visión cognitiva (Charreaux y Desbrières, 
1998). La primera considera a la empresa un sistema de relaciones contractuales, la empresa se define como un "nudo de contratos" entre los individuos (Coriat y Weinstein, 2010). Esta visión se basa en los supuestos de la teoría económica del comportamiento racional ${ }^{8}$ y la búsqueda del óptimo de Pareto por el método de equilibrio (en situación de información imperfecta) (Coriat y Weinstein, 2010). Sin embargo, debido a las asimetrías de información y conflictos de interés, la maximización del valor puede ser difícil o imposible. Por ello, la visión contractual se asocia con una visión restrictiva o negativa, pues la fuente de la eficiencia es por lo general disciplinaria (Charreaux, 2011). La segunda visión de la gobernanza se basa en diversas teorías cognitivas de la empresa9. Se desarrollan sobre la base de supuestos de comportamiento, basados en la racionalidad limitada y la teoría del conocimiento, así como del aprendizaje individual y colectivo en oposición al método de equilibrio. La empresa se define como un "nudo de competencias" (Coriat y Weinstein, 2010). Según Coriat y Weinstein (2010), estas dos visiones están entrelazadas y uno de los principales retos de la teoría de la empresa consiste en determinar cómo se articulan estas dos dimensiones.

El estudio del gobierno corporativo ayuda a entender la creación y distribución de valor en la organización. Por lo tanto, se espera que cualquier organización produzca, a través de la cooperación, un superávit en relación con los recursos consumidos y distribuya este excedente a fin de mantener la sostenibilidad de la organización (Charreaux, 2011). En el caso de grupos cooperativos agrarios, se mostrará más adelante la evolución entre los dos modos de gobernanza a la luz de la visión contractual de la empresa (modo asociativo y modo accionarial). Esto permitirá entender la complejidad de las interacciones de estos dos modos en los grupos cooperativos agrarios, así como resaltar la racionalidad del comportamiento que dio lugar a la creación de las cooperativas agrarias.

Al presentar un modo híbrido de gobernanza, entendemos que la creación de valor en grupos cooperativos reposa sobre las competencias ofrecidas, incluyendo las relaciones de cooperación de larga duración para ciertos empleados o miembros. En este sentido, la

8 Teoría de la agencia, la teoría de los costos de transacción, y la teoría de los contratos incompletos y derechos de propiedad (Coriat y Weinstein, 2010).

9 La teoría del comportamiento, la teoría evolutiva, las teorías de aprendizaje organizacional, y las teorías de los recursos y competencias (Coriat y Weinstein, 2010). visión contractual de la formación de valor proporciona un proceso limitado de creación de valor de los grupos cooperativos. Para entender este proceso, es necesario recurrir a las teorías cognitivas de la empresa (Charreaux, 2011). Ellas conducen a asignar una importancia primordial del desarrollo de competencias y a las capacidades de las empresas para innovar. Por lo tanto, la creación de valor en los grupos cooperativos dependerá de la fuente de las competencias de la organización, concebida como un conjunto coherente (Teece, Rumelt, Dosi y Winter, 1994), que deriva su carácter distintivo de su capacidad para crear conocimiento y, así, que sea rentable de manera sostenible. Una primera parte de nuestro análisis utiliza los conceptos forjados por la visión contractual de la empresa, el modo de crear valor para los accionistas y de manera asociativa.

Las cooperativas tradicionales funcionan bajo el modo de gobernanza asociativa según los principios cooperativos antes mencionados (Gianfaldoni y Richez-Battesti, 2008). Sin embargo, el surgimiento de grupos cooperativos se acerca más a un modo de gobernanza accionarial. En la siguiente sección, se demuestra cómo la gobernanza de los grupos cooperativos se encuentra entre el modo de gobernanza asociativo y accionarial.

\section{Gobernanza cooperativa: del modo asociativo al modo accionarial}

A raíz de los cambios estructurales en las cooperativas agrarias, se podría esperar que estas empresas pasen de un modo asociativo a un modo accionarial, al mismo tiempo que de un modelo de cooperativa tradicional a un modelo de grupo cooperativo. De hecho, ahora se enfrentan a estos dos enfoques para la creación de valor. Esto implica dos formas diferentes de definir la empresa y sus objetivos que aquí se presentan.

\section{El modo asociativo}

En el modo asociativo las empresas tienen como objetivo - a través de la cooperación de los diferentes actores- producir un excedente de recursos y distribuirlos a fin de mantener la sostenibilidad de la organización (Charreaux y Desbrières, 1998; Charreaux, 2003). Por lo tanto, el modo de asociación no se centra únicamente en el análisis de la relación de los accionistas con los dirigentes, sino que también abarca todas las relaciones de la empresa con sus grupos de interés: empleados, clientes, proveedores, acreedores, gobiernos $y$, 
en general, su entorno. El estudio de la gobernanza ha permitido renovar y ampliar el análisis sobre el desempeño de las empresas de la economía social y solidaria, tales como las mutuales y las cooperativas (Charreaux, 2000; Gianfaldoni y Richez-Battesti, 2008). En el caso de las cooperativas agrarias, son empresas cuyo objeto social es una extensión de las actividades de los agricultores, propietarios y clientes. Según el artículo L521-1 del Código Rural, las cooperativas agrarias tienen como objetivo el uso común por los agricultores de todos los medios, con el fin de facilitar o desarrollar su actividad económica, mejorar o incrementar los resultados de esta actividad.

La diferencia entre las cooperativas y las empresas privadas está en que el objetivo de las cooperativas es servir a sus miembros a través de la distribución de los beneficios. Las cooperativas se basan en valores y principios de solidaridad y democracia. Así, la relación con los miembros se basa en los principios de la solidaridad en lugar de la ganancia económica. Los mecanismos de incitación también se basan en la participación en la toma de decisiones y el reconocimiento de la lealtad (rebajas, descuentos por fidelidad) de los asociados. También se hace hincapié en la importancia del papel de financiación de los bancos en los grupos cooperativos (Gianfaldoni y Richez-Battesti, 2008; Charreaux, 1999), pues la relación con los bancos ayuda a reducir la asimetría de la información, en contraste con las empresas privadas.

En este modo de gobierno, la empresa es analizada como un nudo de múltiples contratos entre los diferentes grupos de interés que buscan maximizar el valor de las partes interesadas. Por lo tanto, el aspecto preferido de creación de valor es reducir los costes de agencia relacionados a los conflictos de interés entre los diversos actores de la empresa. Estos costos pueden aparecer debido a la separación entre la propiedad y el control en las grandes organizaciones; por ejemplo, el costo de la toma de decisiones cuando hay muchos grupos de interés en la empresa. Según Gianfaldoni y Richez-Battesti (2008), este modo de gobierno es más complejo, ya que incluye a todos los interesados y en la literatura corresponde a una lógica de control interno basado en los mecanismos institucionales, tales como el consejo de administración, la asamblea general, los comités o las comisiones (Hyafil, 1997). En este modo de gobernanza, se comparte el poder y control entre los socios (los accionistas o socios, los clientes, los empleados, los dirigentes, etc.).
Por lo tanto, la eficacia de este modo de gobernanza depende del consenso entre los diferentes actores de la organización. Para la definición del modo de gobernanza asociativa privilegiamos la de la Organización para el Comercio y el Desarrollo Económicos (OCDE, 2004):

La gobernanza se refiere a la relación entre la gestión de una empresa, su consejo, sus accionistas y otros grupos de interés. (...) también determina la estructura por la que se definen los objetivos de una empresa, así como los medios necesarios para lograr y asegurar los resultados (p. 23).

Se adopta esta definición ya que el campo de análisis que se considera importante de la gobernanza corporativa es particularmente adecuado para el estudio de las cooperativas, ya que toma en cuenta los diferentes grupos de interés de la empresa. Esta definición también fue utilizada por el IFA (2006), en su estudio sobre la gobernanza de las empresas cooperativas y mutuales, considerando que este tipo de empresa no solo debe maximizar la rentabilidad financiera, sino que debe también satisfacer principalmente las necesidades de los miembros/clientes (principio de la doble calidad) que participan simultáneamente en la dirección de la empresa y se benefician de los servicios ofrecidos.

\section{El modo accionarial}

En el modo accionarial, el propósito de la gestión empresarial es maximizar la riqueza del accionista mediante la alineación de la conducta de los dirigentes con los objetivos de los accionistas, a través de los incentivos monetarios y de mecanismos internos y externos de control (Caby y Hirigoyen, 2005). Los incentivos financieros son como las recompensas, bonificaciones y salarios indexados al rendimiento, stock-options y decisiones de despido basadas en el rendimiento (Jensen y Murphy, 1990), cuyo objetivo es resolver el problema de la agencia en relación con la compensación de los dirigentes. Los mecanismos de control son principalmente externos y se dan a través del mercado financiero (Moerland, 1995). Estos son: la geografía del capital, el consejo de administración, la política de endeudamiento, los mercados financieros, el mercado de trabajo y la competencia de otras empresas (Caby y Hirigoyen, 2005). El control interno está representado por el consejo de administración, pero a diferencia 
del modo de gobernanza asociativo su composición está correlacionada con la participación. También se encuentra la presencia de consejeros independientes (Tifafi y Dufour, 2006). El enfoque de la creación de valor para los accionistas apoya el crecimiento de las inversiones (el objetivo de los dirigentes) y asegura la viabilidad financiera (el objetivo de los accionistas). Entre las características que distinguen el modo de gobernanza accionarial, se identifica el papel de los mercados de capital en la financiación (Allen, 1993; Hyafil, 1997). Las principales características de estos dos modos de gobernanza se resumen en la tabla 1 .

En cuanto a las cooperativas agrarias, su transformación en grupos cooperativos las ha llevado a buscar, en cierta medida, el valor del accionista. El riesgo de esta evolución es que la búsqueda de valor asociativo se desvanece poco a poco frente a la búsqueda de valor para los accionistas. Este nuevo modo de gobernanza ya no estaría al servicio del asociado, ni siquiera del accionista, sino de la empresa o de los dirigentes. En conclusión, la asociación del modo de gobernanza asociativo y el modo de gobernanza accionarial en los grupos cooperativos agrarios generó la aparición de un modo híbrido de gobernanza.

\section{El modo de gobernanza híbrido de los grupos cooperativos agrarios}

En esta sección se analizan las consecuencias del modo hibrido de gobernanza para las cooperativas agrarias. Entre los cambios significativos en el modo de gobierno, se toman las características anteriormente presentadas con el fin de describir el modo híbrido de la gobernanza de los grupos cooperativos agrarios en Francia. De acuerdo con el funcionamiento de los grupos de cooperativas, las cooperativas tradicionales se identifican perfectamente con una lógica interna de modo de gobernanza de tipo asociativo. Esta lógica está representada por la pareja presidente-director general, el equipo de dirección-consejo de administración, y la capacitación, la información y la participación de las distintas partes interesadas en el proceso de toma de decisiones internas de la organización (como gerentes, socios, empleados, clientes externos, otras cooperativas y autoridades públicas). Sin embargo, el desarrollo de grupos cooperativos se caracteriza en la literatura por la transición de un modo de gobernanza asociativo a un modo híbrido de gobernanza (Côté, 2006; Draperi, 2007; Gianfaldoni y Richez-Battesti, 2008; Forestier y Mauget, 2000; Richez-Battesti, 2006).

Tabla 1

Las características de los modos de asociación y de gobierno de los accionistas

\begin{tabular}{|c|c|c|}
\hline $\begin{array}{l}\text { Modos de gobernanza } \\
\text { características }\end{array}$ & $\begin{array}{l}\text { Modo asociativo } \\
\text { cooperativas }\end{array}$ & $\begin{array}{l}\text { Modo accionarial } \\
\quad \text { filiales }\end{array}$ \\
\hline \multirow[t]{2}{*}{$\begin{array}{l}\text { Definición del sistema } \\
\text { de gobernanza y objetivo }\end{array}$} & $\begin{array}{l}\text { Conjunto de mecanismos para mantener el nudo de } \\
\text { contratos entre todas las partes interesadas. }\end{array}$ & $\begin{array}{l}\text { Conjunto de mecanismos para mantener el nudo } \\
\text { de contratos entre accionistas y dirigentes. }\end{array}$ \\
\hline & Maximizar el valor asociativo. & Maximizar el valor accionarial. \\
\hline Forma organizacional & $\begin{array}{l}\text { Cooperativas, mutuales: adhesión concentrada, parti- } \\
\text { cipaciones cruzadas (principio de la doble calidad). }\end{array}$ & $\begin{array}{l}\text { Sociedades de accionistas: propiedad difusa (riesgo } \\
\text { de toma de control hostil en el mercado). }\end{array}$ \\
\hline Estructura del poder & $\begin{array}{l}\text { Cogestión de todos los interesados (principio de una } \\
\text { persona-un voto), la estrategia de control a largo } \\
\text { plazo basada en los valores de solidaridad, igualdad y } \\
\text { transparencia. }\end{array}$ & $\begin{array}{l}\text { El poder legítimo de control es de los accionistas, } \\
\text { control a corto plazo sobre la rentabilidad financiera. }\end{array}$ \\
\hline Fuentes de financiación & Bancos. & Mercado de valores. \\
\hline Los mecanismos de control & $\begin{array}{l}\text { Lógica interna: consejos de administración, } \\
\text { asambleas generales, consejo de vigilancia, comités, } \\
\text { comisiones. }\end{array}$ & $\begin{array}{l}\text { Lógica externa: consejo de administración que está } \\
\text { compuesto por accionistas según la propiedad del capi- } \\
\text { tal y la presencia de administradores externos. }\end{array}$ \\
\hline \multirow[t]{3}{*}{ Los mecanismos de incitación } & $\begin{array}{l}\text { Incentivos colectivos derivados de la participación en } \\
\text { la toma de decisiones. }\end{array}$ & $\begin{array}{l}\text { Mecanismos de compensación de los dirigentes relacio- } \\
\text { nados con el desempeño económico. }\end{array}$ \\
\hline & $\begin{array}{l}\text { Beneficios para los grupos de interés (descuentos, } \\
\text { rebajas, principio de a-capitalismo) y el trabajo exclu- } \\
\text { sivo con los adherentes (principio de exclusividad). }\end{array}$ & $\begin{array}{l}\text { Incentivo para controlar vinculado a la posibilidad } \\
\text { de las ganancias sobre el capital y derecho sobre las } \\
\text { reservas. }\end{array}$ \\
\hline & & $\begin{array}{l}\text { Disciplina de mercado: la competencia de bienes y } \\
\text { servicios, capitales y mano de obra. }\end{array}$ \\
\hline
\end{tabular}


Este modo representa la convergencia entre los dos modos de gobernanza: el accionarial cuya adopción es necesaria en el marco de un contexto cada vez más competitivo, y el asociativo que caracteriza tradicionalmente este tipo de empresas (Koulytchizky y Mauget, 2003). De hecho, en los grupos cooperativos se evidencia una dilución de los principios cooperativos originales a causa del cambio de forma organizacional. Los nuevos propietarios son, en primer lugar, los asociados de la cooperativa tradicional, pero también los accionistas-inversores de las filiales del sector privado. El valor a maximizar no es ni asociativo, ni accionarial: es el valor del grupo. El objetivo es mantener la nueva estructura. Tal enfoque propuesto por Charreaux y Desbrières (1998) supone que la relación entre la organización y las partes interesadas se co-construye con el fin de maximizar el valor del grupo, y no solamente con el fin de tener relaciones mercantilistas. Por lo tanto, las restricciones legales se vuelven más flexibles. Por ejemplo, en un grupo cooperativo, el exclusivismo y la libre adhesión no son respetados en las filiales privadas. Entre las características del modo de gobernanza híbrido, la financiación de los grupos cooperativos es realizada por el mercado de capitales. Los mecanismos de control y de incitación cambian hacia un modo de gobernanza accionarial. En este modo de gobernanza híbrido coexisten diversos lugares de control en distintos niveles, lo cual puede generar una desconexión del control de las filiales privadas de la cooperativa. La disciplina de mercado también participa a través de la evolución de los precios de las acciones, el fortalecimiento de la disciplina de los dirigentes por el mercado de productos, las regulaciones y las reestructuraciones.

Por último, encontramos el establecimiento de mecanismos de compensación que incentivan a los dirigentes y empleados (incluyendo asesores agrarios). Con respecto al debilitamiento de los valores y principios cooperativos, el desarrollo de grupos cooperativos agrarios impactó negativamente los valores y los principios tradicionales de las cooperativas. La solidaridad se tornó selectiva y el principio de igualdad se convierte en capital mediante la introducción de un precio para cada servicio ofrecido por la cooperativa (Côté, 2001). Si nos referimos a los principios, el a-capitalismo es por completo eliminado en el modo híbrido de gobernanza. De hecho, el método de pago es el mismo que si el agricultor es accionista de una empresa privada. La democracia cooperativa sigue siendo válida solo dentro de la empresa matriz, pero el poder de las filiales depende de la proporción de capital que representan.
Finalmente, el principio de libre adhesión se puede ver modificado en los grupos cooperativos en beneficio de los intereses de las filiales privadas (Koulytchizky y Mauget, 2001). Las consecuencias de la creación de grupos cooperativos se sintetiza en la tabla 2. Esta incorpora los cambios experimentados por las cooperativas agrarias francesas en la estructura organizacional y la identidad cooperativa (valores y principios; Koulytchizky y Mauget, 2001).

Tabla 2

Características del modo híbrido de gobernanza en los grupos cooperativos agrarios

\begin{tabular}{|c|c|}
\hline $\begin{array}{l}\text { Modo de gobierno } \\
\text { especificaciones }\end{array}$ & $\begin{array}{c}\text { Modo híbrido } \\
\text { grupos cooperativos agrarios }\end{array}$ \\
\hline $\begin{array}{l}\text { Definición del sistema } \\
\text { de gobierno y objetivo }\end{array}$ & $\begin{array}{l}\text { Conjunto de mecanismos para mantener } \\
\text { el grupo Cooperativa. } \\
\text { Objetivo: maximizar el valor del grupo. }\end{array}$ \\
\hline $\begin{array}{l}\text { Forma de la empresa } \\
\text { y de los accionistas }\end{array}$ & $\begin{array}{l}\text { La coexistencia de formas de negocio: } \\
\text { sociedad cooperativa empresa privada }+ \\
\text { local de la lista, a la cabeza del grupo o } \\
\text { holding filial. } \\
\text { La disociación de la identidad del cliente } \\
\text { y unirse a la propiedad cooperativa en } \\
\text { subsidiarias. }\end{array}$ \\
\hline Estructura de poder & $\begin{array}{l}\text { El principio democrático } 1 \mathrm{voz}=1 \\
\text { hombre se puede cumplir en la empre- } \\
\text { sa matriz, pero el poder de filiales está } \\
\text { generalmente en proporción al capital de } \\
\text { cada socio. } \\
\text { La pérdida de la identidad cooperativa = } \\
\text { cambio de valores. }\end{array}$ \\
\hline $\begin{array}{l}\text { Fuentes de } \\
\text { financiamiento }\end{array}$ & $\begin{array}{l}\text { Recaudación de fondos a través de las } \\
\text { explotaciones o de mercado erudito. }\end{array}$ \\
\hline $\begin{array}{l}\text { Los mecanismos } \\
\text { de control }\end{array}$ & $\begin{array}{l}\text { Lógica interna para las sociedades coope- } \\
\text { rativas y lógica externa para las filiales. }\end{array}$ \\
\hline \multirow[t]{3}{*}{$\begin{array}{l}\text { Los mecanismos } \\
\text { de incitación }\end{array}$} & $\begin{array}{l}\text { Incentivo para crear valor en la red de } \\
\text { cooperación y ascenso a la cabeza y los } \\
\text { accionistas (una desviación del principio } \\
\text { del capitalismo). }\end{array}$ \\
\hline & $\begin{array}{l}\text { La disciplina de mercado: criterios de } \\
\text { selección de las actividades relacionadas } \\
\text { con la lógica de la rentabilidad ajustada } \\
\text { al riesgo de mercado. La competencia en } \\
\text { bienes y servicios, capital y trabajo. }\end{array}$ \\
\hline & $\begin{array}{l}\text { La implementación de los acuerdos de } \\
\text { compensación de incentivos para directi- } \\
\text { vos y empleados. }\end{array}$ \\
\hline
\end{tabular}

Nota. Elaboración propia 
Es posible deducir de este cuadro que las consecuencias de la existencia de un modo híbrido de la gobernanza en las cooperativas agrarias son de dos tipos: en primer lugar, en cuanto a los mecanismos de control y de incitación; $y$, luego, en el debilitamiento de los valores y principios cooperativos.

\section{La adopción de un modelo híbrido genera una crisis de identidad}

El paso de las cooperativas a un modelo de gobernanza híbrido se traduce en una serie de efectos negativos sobre la relación entre la cooperativa y sus miembros. Estos efectos se analizan a través de la descripción de la crisis de identidad de los grupos cooperativos realizado por Côté (2001). De esta manera, se distinguen tres efectos principales, y luego se ilustran estos efectos usando ejemplos en grupos cooperativos agrarios franceses.

\section{La crisis de identidad de los grupos cooperativos agrarios}

La literatura sugiere varios efectos negativos relacionados con el surgimiento de grupos cooperativos en diferentes sectores de la economía. En el sector agrícola, en particular, Forestier y Mauget (2001) describen tres efectos negativos de la creación de grupos cooperativos: el distanciamiento, la complejidad y la confusión.

El primer efecto negativo es el distanciamiento. El desarrollo de los grupos cooperativos ha llevado a un aumento en el tamaño de las cooperativas mediante la creación de grupos con miles de asociados. Según Draperi (2007), algunas cooperativas se han alejado de sus asociados constituyendo holdings financieros, combinando estructuras de la economía solidaria con empresas privadas y repartiéndose entre los intereses de los asociados y de los accionistas de las empresas privadas. Los asociados viven así un efecto de distanciamiento de las estructuras de toma de decisiones de la cooperativa. Este distanciamiento esta relacionado con la anonimización de los asociados, pues ya no conocen los dirigentes de la cooperativa. Los miembros de un grupo cooperativo se enfrentan a una empresa desconocida (su historia, su estrategia, la gestión), y no dudan en buscar ofertas más ventajosas, incluso fuera de la cooperativa, en particular en los sectores caracterizados por una alta volatilidad de los precios de las materias primas (como el sector de cereales).
Los asociados ya no son solamente miembros de una cooperativa. Se convierten en clientes y la satisfacción de sus necesidades no es una meta final, sino un paso necesario hacia el incremento del valor accionarial.

Un segundo efecto negativo es la complejidad. Los grupos cooperativos tienen ahora organigramas complejos. Esto en cuanto combinan los organigramas de las asociaciones con los organigramas de empresas comerciales, y se vuelven difusos y difíciles de entender para todos los asociados, lo cual refuerza el efecto de lejanía antes mencionado. De hecho, Thériault (1997) considera que el nuevo modelo cooperativo es más una asociación de personas "ficticia", ya que el participante se convierte en un cliente (la racionalidad utilitaria y comportamiento consumista), el cual no es capaz de valorar la naturaleza de la relación con su cooperativa (Côté, 2007). La pregunta que surge es el sentido de la acción colectiva, el sentido de la cooperativa. De hecho, el aumento en el número de asociados facilita el comportamiento de pasajero clandestino (free rider), mediante el cual un asociado quiere sacar provecho de una acción colectiva sin aportar nada (Olson, 1965). Cada vez es entonces más difícil diferenciar una cooperativa de sus competidores en el sector privado.

El tercer efecto negativo de la aparición de grupos de cooperación en el sector agrícola es la confusión. Los asociados se enfrentan a dos formas de distribución de ingresos: una distribución de ingresos relacionada con la actividad de producción (forma asociativa), y una distribución de los ingresos relacionada con la propiedad del capital (forma capitalista). Esta mezcla de formas asociativa y capitalista es percibida por los asociados como una transformación de sus acciones y sus derechos de uso de la cooperativa en capital de una sociedad anónima. Están asistiendo a un cuestionamiento del modelo cooperativo que se puede resumir en una pérdida de relación entre la cooperativa y sus asociados. Para que este modelo híbrido funcione, las cooperativas tienen que encontrar la manera de fortalecer esa relación para garantizar su sostenibilidad.

\section{Ilustración de los efectos negativos de los grupos cooperativos agrarios en Francia}

Los tres efectos negativos destacados por Côté (2001), presentados en la sección anterior, se pueden ilustrar en el caso de los grupos cooperativos franceses a través de varios ejemplos. Deroy y Thénot (2012), en su estudio de caso de un grupo cooperativo francés, muestran 
cómo el modelo de grupo cooperativo es reconocido en su calidad de generador de distanciamiento, y esto lo demuestran a través de un ejemplo de la comunicación entre los dirigentes y los asociados. El aumento del número de asociados es una de las principales causas del distanciamiento (Vargas-Prieto, 2013). Del mismo modo, si se tienen en cuenta los principales grupos cooperativos en Francia, es posible ver cómo el gran tamaño en el número de asociados afecta la organización de la toma de decisiones, alejándose de los productores (Transrural, 2004). Además, el aumento de tamaño favorece la multiplicación de los intermediarios, lo que genera la pérdida del poder de los asociados (Rastoin, 2000; Transrural, 2004; Zevi y Monzón-Campos, 1995). En efecto, en un contexto de cooperativa tradicional, en la que cada asociado conocía personalmente al menos uno de los directores, les daba la impresión de tener un poco de poder, en el sentido en que podían jugar con esta relación para influir de manera amigable en los administradores (Chiffoleau, 1999). En los grupos cooperativos, los agricultores se sienten anónimos y objetos de beneficio para estas grandes empresas ahora desconocidas (Forestier y Mauget, 2001). Una consecuencia de este efecto es la falta de participación de los miembros en la gobernanza de las cooperativas (Champagne, 1998; Couret, 2002; Côté, 2000).

Deroy y Thénot (2012) también muestran cómo "la estructura del grupo es reconocida mundialmente como sinónimo de complejidad (...) debido a las características específicas de su estructura jurídico-administrativa y financiera" (p. 426). De hecho, el papel de los miembros de los grupos cooperativos varía: algunos grupos limitan la participación de sus asociados a la cooperativa tradicional (a través del consejo de administración y de los dirigentes), otros ubican los asociados en las filiales principales del grupo (los dirigentes de la cooperativa son administradores de las filiales y los presidentes son reclutados dentro de los asociados). Por último, los asociados también pueden tener una participación en la holding (a través de los dirigentes de la cooperativa), pero no en las filiales: los agricultores pueden entonces participar en la dirección a través de sus inversiones capitalistas en acciones de las filialesholdings (Duchamp y Mauget, 2012). Uno de los efectos de esta complejidad se refleja en los mecanismos de fijación de precios, tal como se manifiesta en el testimonio de un agricultor:

Es cada vez más difícil de participar en el establecimiento de los precios, nunca se sabe a qué precio se venden ya que primero uno recibe un anticipo y un precio de depósito y posiblemente uno recibe un saldo adicional o un reembolso el año siguiente. Lo mismo para la compra de productos hechos en la cooperativa porque los descuentos y el precio se establecen de acuerdo con el compromiso del agricultor, la fecha de compra, de venta (Transrural, 2004, p. 8).

Esta complejidad contribuye a la confusión de las estructuras generales de los grupos cooperativos, en la medida en que el proceso de la aparición de filiales en las cooperativas agrícolas ha avanzado (Filippi y Triboulet, 2003). La figura 2 presenta el esquema genérico de la organización de un grupo cooperativo. Se ilustra la complejidad que enfrentan los miembros.

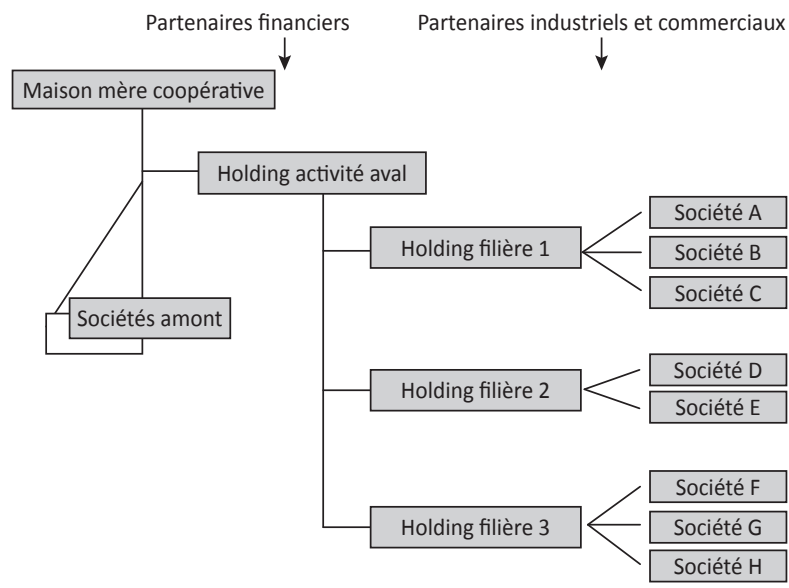

Figura 2. Diagrama genérico de la organización de un grupo cooperativo. Tomado de "De la Coopérative Au Groupe Coopératif Agro-Alimentaire, 1ere Partie: de La Valeur pour l’usager à La Valeur pour l'actionnaire?", por M. Forestier y R. Mauget, 2000, en Recma, 78.

Por último, el aumento de los grupos cooperativos, de forma concomitante con el efecto de la complejidad, hace que se afiance el distanciamiento y la confusión entre la lógica capitalista y la lógica asociativa. Este efecto de confusión se encuentra principalmente en las diferentes modalidades de repartición de ganancias de las filiales para el beneficio de los miembros. De hecho, los ingresos del miembro pueden provenir de dos fuentes diferentes:

- Ingresos como usuario, con la distribución de los ingresos relacionados con la actividad. En este caso, la cooperativa se interpone entre la filial y sus miembros (Forestier y Mauget, 2001). 
- Ingresos como accionista directo o indirecto en las filiales (Forestier y Mauget, 2000; Koulytchizky y Mauget, 2003). En este caso, la cooperativa desaparece detrás de su filial (Forestier y Mauget, 2001).

La crisis de identidad que afectó a los grupos cooperativos agrarios franceses plantea preguntas sobre el mantenimiento de las relaciones con los asociados y cómo se refleja en el funcionamiento de la cooperativa. Según Côté $(2007 ; 2009)$, el reto para los grupos cooperativos agrarios es mostrar que pueden generar, con sus acciones económicas, resultados portadores de valor agregado para sus asociados con respecto a las empresas privadas. Por tanto, es posible asumir que las cooperativas agrarias tratarán de volver a restablecer la relación con sus asociados.

\section{Conclusión}

Se ha demostrado cómo la aparición de grupos de cooperación ha permitido a las cooperativas agrarias adaptarse a los cambios económicos y garantizar así su desarrollo. Pero esto no deja de tener consecuencias para la relación cooperativa-asociado. El cambio en la estructura de las cooperativas agrarias tiene un efecto de distanciamiento, de complejidad y de confusión para los asociados (Forestier y Mauget, 2001). Con la integración de la cooperativa a un grupo empresarial, el asociado tiene la impresión de ser un peón más en un juego económico cuyas reglas están más allá de su alcance (Nicolas, 1993). Hoy en día, la cooperativa se encuentra en un modelo difuso que combina los modos de gobernanza accionarial y asociativo, lo que es difícil de entender para los asociados. Se sienten alejados de los centros de decisión y les resulta difícil identificarse con estas nuevas estructuras (Duvaleix, Cordier y Hovelaque, 2003; Forestier y Mauget, 2001).

\section{Referencias}

Allen, F. (1993). Strategic Management and Financial Markets. Strategic Management Journal, 14, 11-22.

Baron, C. (2003, juin). La gouvernance : débats autour d'un concept polysémique. Revue Droit et Société, 54, 329351.

Bélis-Bergouignan, M. C. y Corade, N. (2008). Fusion des coopératives vinicoles et ancrage territorial. Economie régionale et urbaine, 1, 43-68.

Berle, A. y Means, A. (1932). The Modern Corporation and Private Property. New York: Harcourt, Brace \& World.
Caby, J. y Hirigoyen, G. (2005, septembre-octobre). La valeur partenariale : un outil de gouvernance. La Revue du Financier, 155, 27-41.

CFCA. (1999). Lévolution du secteur coopératif agricole, document de travail. Documento de trabajo.

Champagne, P. (1998). Les administrateurs de coopératives agricoles sont-ils indispensables? RECMA-Revue Internationale de l'Economie Sociale, 269, 32-45.

Chandler, A. (1989). Strategy and Structure: Chapters in the History of the American Industrial Enterprise. Cambridge, MA: MIT Press.

Charreaux, G. (1997). Le gouvernement des entreprises. Paris: Economica.

Charreaux, G. (1999). La théorie positive de l'agence : lecture et relectures. En G. Kœnig (coord.), De nouvelles théories pour gérer l'entreprise du XXIe siècle. Paris: Economica.

Charreaux, G. (2000). La théorie positive de l'agence : positionnement et apports. Revue d'Économie Industrielle, Programme National Persée, 92, 193-214.

Charreaux, G. (2003). Le Gouvernement D’entreprise. En J. Allouche (Coord.), Encyclopédie Des Ressources Humaines (pp. 628-640). Paris: Vuibert.

Charreaux, G. (2011). Quelle théorie pour la gouvernance? De la gouvernance partenariale à la gouvernance cognitive. Cahier du Fargo N. ${ }^{\circ} 1110402$.

Charreaux, G. y Desbrières, P. (1998, juin). Gouvernance Des Entreprises: Valeur Partenariale Contre Valeur Actionnariale. Finance Contrôle Stratégie, 2, 57-88.

Chiffoleau, Y. (1999). Je ne vais pas aux Ag Et Pourtant J'ai Des Idées : Pour Une Coopération Rurale En Languedoc-Roussillon. Revue des Études Coopératives Mutualistes et Associatives, 273, 27-39.

Cogeca. (2011). Agricultural Co-Operatives in UE, Main Issues and Trends. Brussels: Cogeca-European Agri-Cooperatives.

Colson F. (1990). Difficultés Financières et Viabilité des Exploitations Agricoles. Économie Rurale, 198, 35.

Coop De France. (2010). Poids Économique et Social 2010 de la Coopération Agricole et Agroalimentaire Française. Observatoire Économique Coop De France.

Coriat, B. y Weinstein, O. (2010). Les Théories De La Firme Entre "Contrats » Et "Compétences ». Revue D’économie Industrielle, 129-130, 57-86.

Côté, D. (2000). Les Coopératives et le Prochain Millénaire: Lémergence d'un Nouveau Paradigme Coopératif. Recma, 275-276, 149-166.

Côté, D. (2001). Les Holdings Coopératifs: Typologie, Questionnements et Scénarios de Solutions. En D. Côté (Dir.), Les Holdings Coopératifs: Évolution Ou Transformation Définitive? (pp. 385-402). Bruxelles: De Boeck Université. 
Côté, D. (2006). Agropur: Enjeux et Défis d'une Coopérative à La Recherche d'une Nouvelle Cohésion. Revue Internationale De Cas en Gestion, 4(5), 4-5.

Côté, D. (2007). Fondements d'un nouveau paradigme coopératif (NPC): quels incitatifs pour les acteurs clés? (Working paper). Montréal, HEC.

Côté, D. (2009). Le mode d’organisation coopérative au 21e siècle: un nouveau paradigme coopératif face à la crise identitaire. Revue Internationale de Projectique, 2, 61-84.

Couret, F. (2002). Principe Démocratique et Décision en Coopérative. Recma-Revue Internationale de l'economie Sociale, 285, 13-26.

Deneux, M., Bizet, J. y Dussaut, B. (1999). Lavenir Du Secteur Agro-Alimentaire. Rapport Fait Au Nom de la Commission des Affaires Economiques et du Plan, 83.

Deroy, X. y Thenot, M. (2012). Linteraction des Logiques Coopérative et de Marché: Quelle évolution pour le modèle coopératif agricole français ? Le cas de Champagne Céréales. En M. J. Brassard y E. Molina (Dir.), Létonnant Pouvoir Des Coopératives. Textes Choisis de L'appel International de Propositions (p. 665). Québec: Sommet International des Coopératives.

Draperi, J. F. (2007). Comprendre l'économie sociale. Dunod.

Duchamp, B. y Mauget, R. (2012, janvier). La Course à la Taille, Stratégies de Concentration et d'alliance dans les Coopératives Agricoles Françaises Du Top 20 Avec Comparaison Européenne. Colloque Addes, 24.

Duvaleix, S., Cordier, J. y Hovelaque, V. (2003). Vers un nouvel engagement coopératif dans le secteur laitier: l'économie sociale en mouvement. Revue internationale de l'économie sociale, 288, 37-47.

Eurostaf. (2007). Les Mutations et les Perspectives de Développement des Coopératives Agricoles en France. Etude Eurostaf réalisé par Filippi, M., Mauget, R., (175). Collection Dynamique des Marchés.

Filippi, M. (2002). Les sociétés coopératives agricoles: entre ancrage territorial et intégration économique. Etudes et Recherche sur les Systèmes Agraires, 33, 25-38.

Filippi, M. D., Frey, O., Triboulet, P. y Vivensang, J. (2006). Bilan des Lois de 1991 et 1992 et gouvernance des groupes coopératifs. Toulouse: Inra-Sad.

Filippi, M. y Triboulet, P. (2003). Modalités d'exercice du pouvoir dans le contrôle mutualiste: le cas des groupes coopératifs agricoles. Cahiers Du Gres, 12.

Forestier, M. y Mauget, R. (2000). De la coopérative au groupe coopératif agro-alimentaire, 1ere Partie: de la valeur pour l'usager à la valeur pour l'actionnaire? Recma, 278, $1-15$.

Forestier, M. y Mauget, R. (2001). De la coopérative au groupe agro-alimentaire. Recma, 279, 60-70.
Fort, F. y Couderc, J. P. (2001). Le terroir: un avantage concurrentiel à l'exportation? Le cas des entreprises agro-alimentaires du Languedoc-Roussillon. Économie Rurale, 264-265, 45-69.

Gianfaldoni, P. y Richez-Battesti, N. (2008, mars). La gouvernance partenariale des banques coopératives françaises. Rapport de Recherche Financé par la Diieses.

Guillouzo, R., Perrot, P. y Ruffio, P. (1999, décembre). Typologie, déterminants et spécificités des alliances dans l'industrie agro-alimentaire française. Cahiers d'Economie et Sociologie Rurales, 52, 23-57.

Hyafil, A. (1997). Corporate governance: une synthèse de la littérature. Cahier de Recherche Hec.

IFA. (2006). Coopératives et mutuelles: un gouvernement d'entreprise original. Rapport du groupe de travail présidé par Etienne Pflimlin dans le cadre de l'Institut Français d'Administrateurs-IFA. La Confédération National du Crédit Mutuel.

Insee. (2010). Lagroalimentaire, de l'industrie au commerce de gros : des activités diversifiées et performantes, 5.

Jensen, M. y Murphy, K. J. (1990). Performance pay and topmanagement incentives. Journal of Political Economy, 98, 225-262.

Koulytchizky, S. y Mauget, R. (2001). Mutations et valeurs dans les groupes coopératifs. En D. Côté (Ed.), Les holdings coopératifs (pp. 77-102). Bruxelles: De Boeck.

Koulytchizky, S. y Mauget, R. (2003). Le développement des groupes coopératifs agricoles depuis un demi-siècle. À la recherche d'un nouveau paradigme. Recma, 287, 1440.

Lesieur, C. (2012). Les statistiques de la coopération agroalimentaire: un état des lieux en 2010. Colloque International «Diversité et durabilité des modèles agricoles coopératifs dans un contexte de crises de la mondialisation», Paris, 6 et 7 Novembre.

Magrini, M., Fares, M. y Filippi, M. (2011, octobre). La signalisation de la qualité chez les petites coopératives agricoles françaises. Revue d'Économie Régionale \& Ur baine, 4, 705-733.

Manoa, J. Y. (2001). Structure de groupe et économie sociale. En D. Côté (Ed.), Les holdings coopératives (pp. 25-52). Bruxelles: De Boeck.

Moerland, P. W. (1995). Alternative Disciplinary Mechanisms in Different Corporate Systems. Journal of Economic Behavior and Organization, 26, 17-34.

Nicolas, P. (1992). La remise en cause de l'organisation coopérative: crise d'identité et législation. Nouvelle Economie et Sociologie Rurales, Série Iaa. Nº Dt 9202, 4. 
Nicolas, P. (1993). Règles et principes dans les sociétés coopératives agricoles françaises: évolution du droit et des pratiques de 1960 à 1992. En A. Zevi y J. L. MonzonCampos (Dir.), Coopératives, marchés, principes coopératifs (pp. 155-185). Brussels: De Boeck Université.

OCDE. (2001). Impliquer les citoyens: l'information, la consultation et la participation du public dans le processus de prise de décision. Note de Synthèse de l'ocde sur la Gestion Publique.

OCDE. (2004). Principes de gouvernement d'entreprise de l'OCDE. Paris: oCDE. Disponible en: http://www.oecd. org/fr/daf/ae/principesdegouvernementdentreprise/ 31652074.PDF

Olson, M. (1965). The logic of collective action. Cambridge, MA: Harvard University Press.

Plane, J. M. (2000). Théorie des organisations. Paris: Les Topos.

Rastoin, J. L. (2000). Une brève histoire de l'industrie alimentaire. Economie Rurale, Numéro Spécial 255-256, 61-71.

Richez-Battesti, N. (2006). Entre banalisation et reconquête de l'identité coopérative: le cas des banques coopératives en France. Communication au Colloque International du Réseau Rulescoop: les Défis du Secteur des Organisations Coopératives et Mutualistes, Brest, 22- 24 Mai.

Rouault, P. (2010). Rapport: analyse comparée de la compétitivité des industries agroalimentaires françaises par rapport à leurs concurrentes européennes. Paris: DIIAA.
Saint-Pierre, D. (2004). La politique culturelle du Québec de 1992 et l'advocacy coalition framework. Canadian Journal of Political Science, 37(03), 561-580.

Spear, R. (2001). Globalisation et stratégies des coopératives. En D. Côté (Dir.), Holdings coopératifs: evolution ou transformation définitive? (pp. 115-138). Brussels: De Boeck.

Teece, D. J., Rumelt, R., Dosi, G. y Winter, S. (1994). Understanding Corporate Coherence. Journal of Economic Behavior and Organization, 23, 1-30.

Theriault, J. Y. (1997). Les figures changeantes de la communauté des sociétaires Desjardins. En B. Lévesque (Dir.), Desjardins, une entreprise et un mouvement? (pp. 135152). Québec: Presses De L’université Du Québec.

Tifafi, F. y Dufour, D. (2006). Une analyse de la présence d'administrateurs indépendants au sein du conseil d'administration: contrôle ou conseil ? Comptabilité, contrôle, audit et institutions. Tunisie: République française.

Transrural. (2004, juin). La coopération agricole en débat. Transrural, 263, 8.

Vargas-Prieto, A. (2013). Le rôle du conseil agricole dans la gouvernance coopérative. Thèse de Doctorat en Sciences Economiques, Université Montesquieu.

Zevi, A. y Monzon-Campos, J. L. (1995). Coopératives, marchés, principes coopératifs. Brussels: De Boeck Université. 
Anexo

Factores de evolución de las cooperativas agrarias en Francia

\begin{tabular}{lll}
\hline \multicolumn{1}{c}{$\begin{array}{c}\text { Factores de cambio } \\
\text { para las cooperativas agrarias }\end{array}$} & \multicolumn{1}{c}{ Consecuencias } & \multicolumn{1}{c}{ Desafíos para el sector cooperativo } \\
\hline $\begin{array}{l}\text { Internacionalización y globalización } \\
\text { de los mercados y los competidores. }\end{array}$ & $\begin{array}{l}\text { Cambio en la estrategia por la necesidad de } \\
\text { reducir costos o diferenciar los productos frente } \\
\text { a la competencia. }\end{array}$ & $\begin{array}{l}\text { Adaptarse al nuevo contexto encontrando } \\
\text { un tamaño óptimo para la reducción de las } \\
\text { restricciones legales (exclusividad, la } \\
\text { territorialidad, el a-capitalismo), sin perder } \\
\text { la identidad cooperativa }\end{array}$ \\
\hline Evolución del consumo en Francia. & $\begin{array}{l}\text { Adaptar el producto a la demanda de los con- } \\
\text { sumidores. }\end{array}$ & $\begin{array}{l}\text { Encontrar recursos financieros para desarrollar } \\
\text { nuevas tecnologías y productos innovadores. }\end{array}$ \\
\hline $\begin{array}{l}\text { Evolución de las limitaciones } \\
\text { reglamentarias. }\end{array}$ & $\begin{array}{l}\text { Disminución de la protección contra caídas } \\
\text { en los precios y la aplicación de la ayuda por } \\
\text { hectárea y no por la producción. }\end{array}$ & $\begin{array}{l}\text { El mantenimiento de las exportaciones agrarias } \\
\text { (ventajas comparativas). }\end{array}$ \\
& $\begin{array}{l}\text { Exigencia de cumplimiento de nuevas normas. } \\
\text { La participación en iniciativas de desarrollo } \\
\text { sostenible. }\end{array}$ & $\begin{array}{l}\text { las normas. } \\
\text { Producir más y mejor en una lógica de desarrollo } \\
\text { sostenible. }\end{array}$ \\
\hline $\begin{array}{l}\text { La evolución del comportamiento necesarios para cumplir con } \\
\text { de los agricultores. }\end{array}$ & $\begin{array}{l}\text { Evolución de los valores cooperativos (pasando } \\
\text { de la igualdad a la equidad): pérdida del vínculo } \\
\text { cooperativa-adherente. }\end{array}$ & $\begin{array}{l}\text { Mantener relaciones con los adherentes: creación } \\
\text { de una estrategia de marketing diferenciada para } \\
\text { los adherentes comprometidos. }\end{array}$ \\
& El aumento de las exigencias de los adherentes. \\
& $\begin{array}{l}\text { Desarrollar competencias que permitan un mejor } \\
\text { acompañamiento de los agricultores (cambiar } \\
\text { asesoramiento técnico a la asesoría integral). }\end{array}$ \\
\hline
\end{tabular}

Nota. Elaboración propia 